\title{
Robustness analysis of a parallel two-box digital polynomial predistorter for an SOA-based CO-OFDM system
}

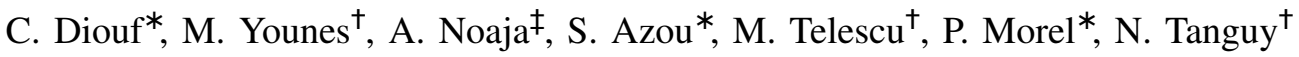 \\ * ENIB / CNRS UMR 6285 Lab-STICC, Brest, France \\ ${ }^{\dagger}$ Univ. de Bretagne Occidentale / CNRS UMR 6285 Lab-STICC, Brest, France \\ $\ddagger$ Military Technical Academy, Bucharest, Romania
}

\begin{abstract}
The linearization performance of various digital baseband pre-distortion schemes is evaluated in this paper for a coherent optical OFDM (CO-OFDM) transmitter employing a semiconductor optical amplif er (SOA). In particular, the benef ts of using a parallel two-box (PTB) behavioral model, combining a static nonlinear function with a memory polynomial (MP) model, is investigated for mitigating the system nonlinearities and compared to the memoryless and MP models. Moreover, the robustness of the predistorters under different operating conditions and system uncertainties is assessed based on a precise SOA physical model. The PTB scheme proves to be the most effective linearization technique for the considered setup, with an excellent performance-complexity tradeoff over a wide range of conditions.
\end{abstract}

Index Terms

Coherent Optical OFDM, Semiconductor optical amplif er (SOA), Digital Predistortion, Linearization, PAPR reduction, Robustness analysis.

\section{INTRODUCTION}

Orthogonal frequency-division multiplexing (OFDM) has been recognized as one of the most promising techniques to support high data rate in next-gen optical communications networks, with some important advantages like simple compensation of linear channel impairments, dynamic bandwidth allocation capability in a multiuser context, and powerful digital signal processor (DSP)-based implementation [1][2][3]. However, a well-known drawback of multicarrier signalling is the high Peakto-Average-Power Ratio (PAPR) [4], which makes OFDM very sensitive to nonlinear devices. Hence, PAPR reduction has been a subject of intense research in the past decade [5], with a wide variety of approaches originally developed for wireless and wireline communications and later investigated for optical OFDM systems [6]. Recently, Amiralizadeh et al. made an important contribution to the modeling and compensation of CO-OFDM transmitter nonlinearity [7] in presence of high PAPR, with a theoretical analysis of the impact of different nonlinear components (DAC, electrical power amplif er, optical modulator) in terms of bit-error-rate (BER) for different clipping ratios. It is also proposed to apply clipping along with digital predistortion to mitigate performance degradation. Some of the present authors have investigated similar problems by considering nonlinear effects originating from optical components, with a special focus on Semiconductor Optical Amplif ers (SOAs) [8][9]. Interesting features such as low cost, large optical bandwidth and small form factor [10] make SOAs an interesting alternative to the high-end EDFAs for some application scenarios [11]. However, as pointed out in our previous study, the use of the SOA as a booster amplif er may introduce strong nonlinear effects such as cross phase modulation (XPM) and four wave mixing (FWM) and degrade the overall system performance. PAPR reduction is required for shaping the envelope dynamics so as to limit the signal distortion but, as in [7], for a better performance improvement this may be combined with some linearization technique for compensating the nonlinear effects inherent to the SOA (which we consider as the main source of nonlinear distortion here). A broad variety of methods have been studied in literature for linearizing optical links or radio-over-f ber $(\mathrm{RoF})$ links over the past few years, with a few approaches being specif cally designed to cope with the nonlinear effects of SOA [12][13]. The various methods can be classif ed into three main groups: optical linearization, electrical analog linearization and electrical digital linearization [14]. A digital baseband predistortion (DPD) is considered throughout this study, which consists in pre-compensating the transmitter nonlinear characteristics based on a black-box behavioral model. Thus irregular characteristics of the transmitter can be counteracted in a f exible way, with a limited knowledge of physical link parameters and at a limited implementation effort. DPD has been the method of choice for power amplif er linearization in microwave wireless communication systems for a long time [15][16]. Numerous formulations exist featuring various block architectures either including or neglecting memory effects. The optical communications community has so far shown caution in importing such methods, but some proof-of-concept studies do exist and the effectiveness of DPD in optical $\mathrm{f}$ ber systems has been reported in a number of studies for different modulation formats (single carrier QAM, OFDM, CDMA) at various data rates and for different system setups/devices. The Memory Polynomial (MP) model [17]-[26], or its generalized formulation 
(GMP) [27][28], is extensively adopted for its good performance. The interest of using a simple look-up-table (LUT) scheme is mentioned in a few references [29][30][31] when the nonlinearity under test can be assumed as memoryless. It is also possible to use box-oriented models, which may be an attractive solution for lowering the implementation complexity while achieving good performance. In [33], it is proposed to combine the advantages of MP and Envelope Memory Polynomial (EMP) in a hybrid parallel structure whereas in [32] the authors study a Hammerstein model, which is composed of a memoryless nonlinearity function followed by a FIR filter. In our previous study [9], we investigated a Filter LUT scheme, which belongs to the augmented Hammerstein family, but with no special attempt to lower the complexity. The present paper tackles this issue which is crucial at optical data rates. A parallel two-box behavioral model is first examined for improving the same SOA-based transmitter, with a static nonlinear function and an MP model and with the objective of keeping the complexity as low as possible (low number of model parameters). It is proposed to design the static block by jointly considering the linearization and PAPR reduction objectives, via a simple constrained polynomial fitting. A second objective is to analyze the robustness of the predistorter in presence of parameter variations in the transmitter. Some of these parameters are physical such as the peak-to-peak voltage of the Mach-Zehnder modulator, optical power and wavelength of the optical signal or bias current for the SOA while others constitute changes in the modulation such as the number of subcarriers. To the best of the authors' knowledge this sort of analysis has not yet been conducted although it is extremely useful in the perspective of meeting future network demands, involving possibly adaptive transceiver parameters [34].

\section{CO-OFDM SYSTEM MODEL}

The following study is based on the coherent optical OFDM system model described in Fig. 1. The setup comprises a transmitter, a Semiconductor Optical Amplifier (SOA) device for boosting transmission reach and performance, and a receiver. Except the digital predistortion block, aiming at counteracting the SOA nonlinearities, the transmitter and receiver architectures are standard with common blocks such as QAM mapping/demapping, serial-to-parallel (S/P)/parallel-to-serial (P/S) conversion, FFT/IFFT transforms, Cyclic Prefix (CP) adding/removing, digital-to-analog (D/A)/analog-to-digital (A/D) conversion, time synchronization and equalization. At the transmitter side electro-optical (E/O) conversion is performed by a Mach-Zehnder modulator (MZM) and linked to the main transmission laser (LD1). A second laser (LD2) is used to perform the coherent detection.

To carry out analyses in interest, the setup is implemented in a Matlab-ADS co-simulation environment. The CO-OFDM transmitter and receiver are modeled in Matlab while the SOA is modeled in ADS Ptolemy using the carrier density rate and optical signal field propagation equations[8]. Optical Amplifier parameters are tuned to realistically fit a $750 \mu \mathrm{m}$ long commercial SOA (INPHENIX-IPSAD1501). Regarding the lasers, the study assumes perfect phase noise compensation and no frequency offset on the receiver side. A standard nonlinear model of the IQ optical modulator is implemented [1], with no imbalance impairment. For the D/A and A/D, a uniform quantization is considered, with a default resolution of 12 bits throughout the manuscript; the effect of the resolution will be investigated in section IV by decreasing its value to 4 bits. An ideal coherent detection is assumed (ideal photodetectors).

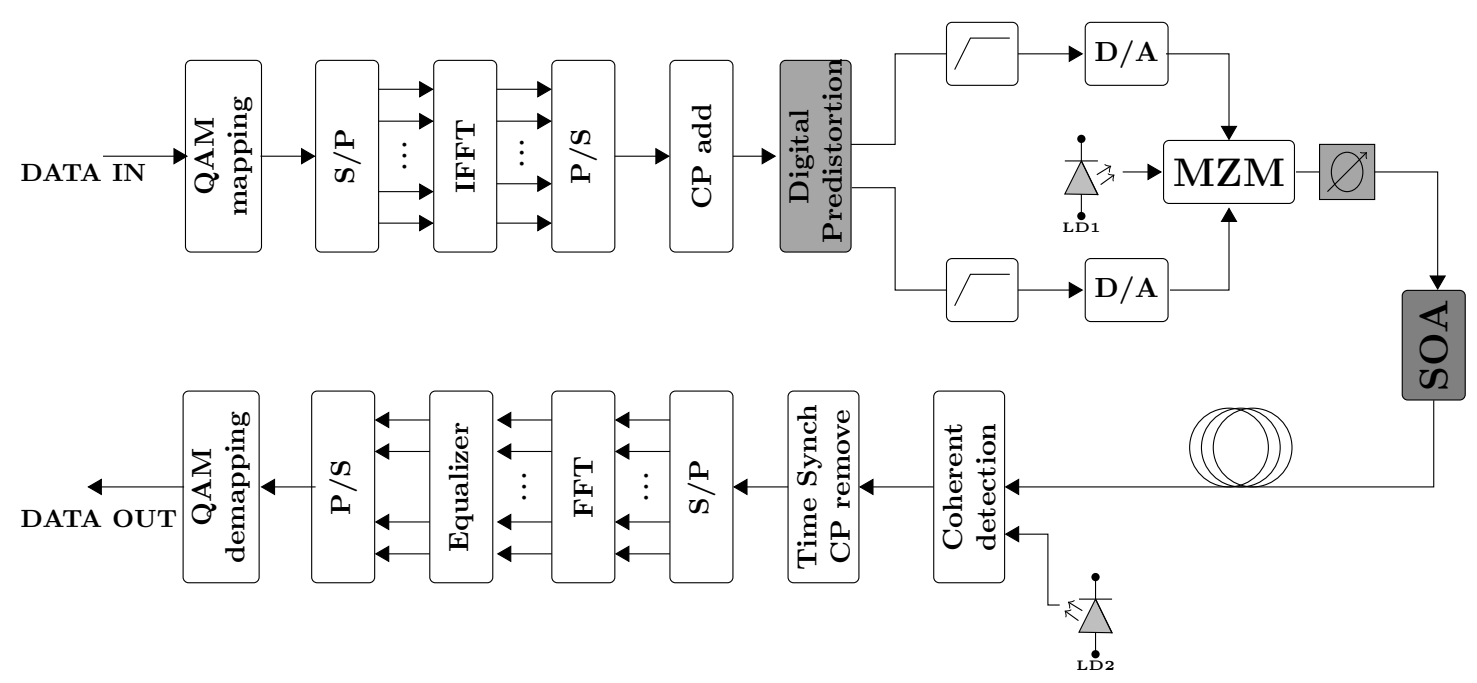

Fig. 1. CO-OFDM structure

As presented in Fig. 1, input data stream is processed by the transmitter to obtain the initial OFDM electrical signal. Hardclipping with a threshold of $12 \mathrm{~dB}$ is applied on the signal in front of DACs just before E/O conversion and the signal at SOA input has its power adjusted via an optical attenuator. Then, the equivalent OFDM optical signal with the SOA model 


\begin{tabular}{|c|c|c|c|c|c|}
\hline Bandwidth: & $20 \mathrm{Ghz}$ & Modulation Format: & 4-QAM & Oversampling factor: & 4 \\
\hline Sampling Time: & $25.6 \mathrm{~ns}$ & Number of subcarriers: & 512 & CP length ratio: & 0.125 \\
\hline$V_{p p}:$ & 8 Volts & Biasing current: & $150 \mathrm{~mA}$ & Alpha factor $\left(\alpha_{H}\right):$ & 3.3 \\
\hline
\end{tabular}

TABLE I

TRANSMISSION SYSTEM PARAMETERS

are provided to the ADS environment for simulations relying on the field propagation equations. Once the simulation has been carried out, the Amplified Spontaneous Emission (ASE) noise sequence is calculated and added to the optical signal which is finally sent in the receiver which computes output data stream (back-to-back evaluation). Throughout the paper, we consider a 17 Gbps transmission with the default parameters presented in Table 1.

The structure and identification techniques of the predistortion systems in this study will be detailed in the following section.

\section{Predistortion StruCtures}

The general concept behind predistortion is to precompensate amplification impairments by distorting the signal injected to the amplifier. The operation is carried out by a predistorter which basically corresponds to an inverse function of device under study. Four polynomial-based structures will be comparatively used to model the SOA inverse function: the static predistorter, the memory polynomial predistorter, the envelope memory polynomial predistorter and the parallel twin boxes predistorter. The memoryless digital predistorter (STATIC, Fig. 2.a) is defined by a direct mapping between the input signal $x$ and the model output signal $y$. The memory polynomial (MP, Fig. 2.b) and the envelope memory polynomial (EMP, Fig. 2.c) are nonlinear models that take account of both nonlinearity orders and memory effects. The parallel twin boxes polynomial predistorter (PTB, Fig. 2.d) is built by associating in parallel a memoryless structure and a MP structure.

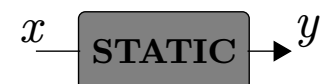

(a)

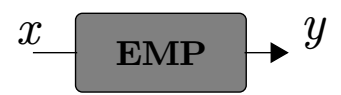

(c)

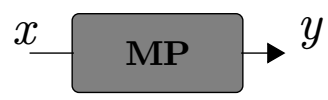

(b)

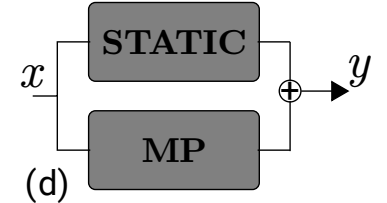

Fig. 2. The four digital predistorters considered in this study: (a) Memoryless predistorter $H_{S T}$, (b) Memory Polynomial predistorter $H_{M P}$, (c) Envelope Memory Polynomial predistorter $H_{E M P}$, (d) Parallel twin boxes predistorter $H_{P T B}$

\section{A. Memoryless polynomial predistorter (STATIC)}

Memoryless systems are very commonly modeled by Look-up-Table (LUT) mappings. We, however, focus on the alternative polynomial modeling approach thanks to its straightforward least square identification process and the lower parametric complexity that it yields. At discrete time $k$, distortion input-output relation is defined as

$$
y[k]=H_{S T}\{x[k]\}=G(|x[k]|) e^{\left(j \phi_{x[k]}+\Delta_{A M / P M}(|x[k]|)\right)} .
$$

where $|x[k]|$ and $\phi_{x[k]}$ denote input electrical magnitude and phase, respectively. $G($.$) and \Delta_{A M / P M}($.$) are two polynomials$ of $|x[k]|$ of $N_{1}$ and $N_{2}$ order; they are respectively described by $\mathbf{a}=\left[a_{0} \ldots a_{N_{1}}\right]^{T}$ and $\mathbf{b}=\left[b_{0} \ldots b_{N_{2}}\right]^{T}$ vectors. SOA amplitudeamplitude distortions are compensated by predistortion gain $G($.$) while SOA amplitude-phase impairments are compensated$ by phase shift $\Delta_{A M / P M}($.$) :$

$$
G(|x[k]|)=\sum_{i=0}^{N_{1}} a_{i}|x[k]|^{i} \text { and } \Delta_{A M / P M}(|x[k]|)=\sum_{i=0}^{N_{2}} b_{i}|x[k]|^{i}
$$

The total number of parameters describing the model is $N_{1}+N_{2}+2$. 


\section{B. Memory Polynomial (MP) and Envelope Memory Polynomial (EMP) predistorters}

MP predistorter, $H_{M P}$, is defined as a memory polynomial model of $N_{3}$ order and $M_{1}$ samples of memory as follows

$$
y[k]=H_{M P}\{x\}=\sum_{i=1}^{N_{3}} \sum_{j=0}^{M_{1}-1} c_{i, j} x[k-j]|x[k-j]|^{i-1} ; \text { i odd }
$$

A total of $\left\lfloor\frac{N_{3}}{2}\right\rfloor \times M_{1}$ parameters is describing the model as only odd orders are actually useful.

$H_{E M P}$ predistorter is a simplified version of the MP predistorter where only the current baseband complex input sample is taken into account:

$$
y[k]=H_{E M P}\{x\}=x[k] \sum_{i=1}^{N_{3}} \sum_{j=0}^{M_{1}-1} c_{i, j}|x[k-j]|^{i-1} ; \text { i odd }
$$

Studied MP, EMP predistorters in the following sections will be free of even nonlinearity orders.

\section{Parallel two boxes digital polynomial predistorter (PTB)}

Finally, the PTB is built on by associating in parallel (Fig. 2c) the memoryless polynomial structure $\left(H_{S T}\right)$ and the MP structure $\left(H_{M P}\right)$. For a discrete input signal $x[k]$, the predistorter output $y[k]$ is given by

$$
\begin{gathered}
y[k]=H_{P T B}\{x\}=H_{S T}\{x\}+H_{M P}\{x\} \\
y[k]=\sum_{i=0}^{N_{1}} a_{i}|x[k]|^{i} e^{\left(j \phi_{x}+\sum_{i=0}^{N_{2}} b_{i}|x[k]|^{i}\right)}+\sum_{i=1}^{N_{4}} \sum_{j=0}^{M_{2}-1} d_{i, j} x[k-j]|x[k-j]|^{i-1} ; \text { i odd }
\end{gathered}
$$

The parametric complexity amounts to $N_{1}+N_{2}+2+\left\lfloor\frac{N_{4}}{2}\right\rfloor \times M_{2}$.

\section{IDENTIFICATION OF BLACKBOX MODELS}

Identification consists in the computation of the considered model coefficients. As predistortion is carried out in electrical domain, identification stimuli are the transmitted electrical signal $x$, just before E/O conversion and the received electrical signal $x_{r}$, just after O/E conversion (Fig. 1). Accordingly, a simulation with a $2^{15}$ 4-QAM symbols input sequence is first performed without predistortion in the transmission chain. Then $x$ and $x_{r}$ signals retrieved from simulation results are forwarded to least squares (LS) based algorithms for each predistorter set of coefficients computation.

\section{A. Constrained static predistorter identification}

Static parameters can be computed by applying a second order polynomial fit on the inverse AM/AM curve (Fig. 3.a). Nevertheless directly applying this method does not always guarantee optimal polynomial coefficients regarding to Error Vector Magnitude (EVM) performance. In fact digital predistortion while compensating, to a certain amount, SOA nonlinearities tends to accentuate impairments resulting from high PAPR; the technique's overall efficiency is hence impacted. To increase EVM performance, the polynomial fitting is enforced to meet a specific constraint point $C\left(x_{c}, y_{c}\right)$ towards large amplitudes. The constraint abscissa $x_{c}$ is based on the signal complementary cumulative distribution function (CCDF) of PAPR; here we consider a value on par with a $12 \mathrm{~dB}$ hard-clipping. The best ordinate $y_{c}^{*}$ is then computed via a golden section search algorithm [35] within an initial interval $\left[0, y_{c}^{i}\right]$, with $y_{c}^{i}<x_{c}$. As can be seen in Fig. 3.b, EVM actually presents a convex variation with respect to $y_{c}$. In Table II default ordinate value $y_{0}$, i.e without constraints, and optimal ordinate $y_{c}^{*}$ are compared for $x_{c}=2$. It can be clearly observed that $y_{c}^{*}$ is decreasing when the reference input power $P_{r e f}$ used for identification increases, thus achieving a crest factor reduction with no need of a separate PAPR reduction block. As a result the constrained model is showing better performance than the default static model, especially when the SOA operates in the saturated regime. An improvement of $3 \%$ can be noticed at $P_{r e f}=-11 \mathrm{dBm}$ while there is practically no improvement when operating close to the SOA linear region $\left(P_{\text {ref }}=-18 \mathrm{dBm}\right)$.

In Fig. 3.c and Fig. 3.d, for $P_{r e f}=-14 \mathrm{dBm}$ and $P_{\text {ref }}=-11 \mathrm{dBm}$ as respective identification reference, we compare constrained and unconstrained model when input optical power $P_{i n}$ is variating from $-27 \mathrm{dBm}$ to $-10 \mathrm{dBm}$. It is observed that the constrained inverse model is offering superior performance all over the power range. Also, as for following PTB predistorters, built up by associating a pure static block and a dynamic block, a constrained memoryless block will be chosen over a classic unconstrained static block. Parallel-Two-Boxes predistortion is presenting higher EVM improvement with optimized static component. 

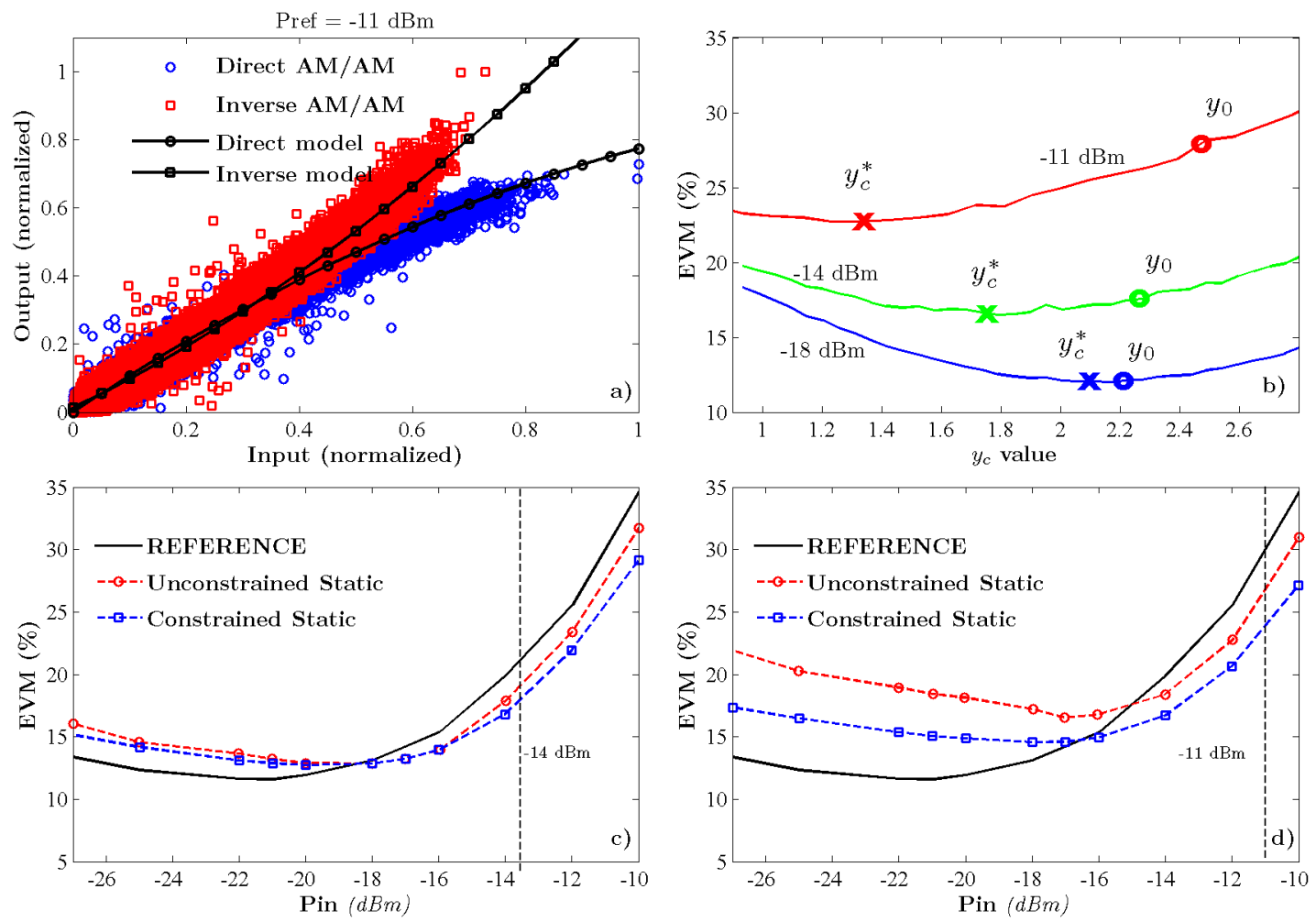

Fig. 3. (a) AM/AM direct and inverse plot, (b) EVM vs $y_{c}$ for $P_{r e f}=-18,-14,-11 \mathrm{dBm}$, (c) STATIC predistortion EVM against input power at $P_{r e f}=-14 \mathrm{dBm}$, (d) STATIC predistortion EVM against input power at $P_{r e f}=-11 \mathrm{dBm}$

\begin{tabular}{|c|c|c|c|}
\hline$P_{\text {ref }}$ & $-18 \mathrm{dBm}$ & $-14 \mathrm{dBm}$ & $-11 \mathrm{dBm}$ \\
\hline Unconstrained ordinate $y_{0}$ & 2.2 & 2.26 & 2.41 \\
\hline Optimal ordinate $y_{c}^{*}$ & 2.1 & 1.75 & 1.62 \\
\hline Reference PAPR (dB) & 8.87 & 8.87 & 8.87 \\
\hline STATIC predistortion PAPR (dB) & 9.27 & 7.8 & 7.23 \\
\hline$\Delta E V M(\%)$ & 0.01 & 1.1 & 3 \\
\hline
\end{tabular}

TABLE II

DEFAULT ORDINATE $y_{0}$ VS OPTIMAL $y_{c}^{*}$ WHEN CONSTRAINED POLYNOMIAL FITTING IS APPLIED; EQUIVALENT PAPR AND EVM IMPROVEMENT

\section{B. Memory Polynomial predistorters identification}

Although they implement nonlinear functions, MP predistorters have a linear input-output relation with respect to model parameters. MP, EMP and PTB will accordingly be identified by an offline LS algorithm which relies on a QR decomposition for better numerical stability [36]. Identification stimuli are the same $2^{15}$ 4-QAM $x$ and $x_{r}$ signals used to compute static model parameters. Identification is carried out for $P_{\text {ref }}=-18,-14$ and $-11 \mathrm{dBm}$.

1) Parametric complexity sweep : Model order and memory depth settings are not known a priori. We therefore proceed to the identification of a series of predistorters with different order and memory depth configuration. Order and depth are varying from 1 to 5, only odd orders being used. Each predistorter is identified and related validation simulations are carried out. In Fig. 4 EVM against optical input power $P_{i n}$ is presented. Results are first shown for $P_{r e f}=-14 \mathrm{dBm}$.

\begin{tabular}{|c|c|c|c|c||c|}
\hline Pin & $-26 \mathrm{dBm}$ & $-20 \mathrm{dBm}$ & $-\mathbf{1 4} \mathbf{~ d B m}$ & $-11 \mathrm{dBm}$ & Complexity \\
\hline \hline STATIC & -1.8 & -0.72 & $\mathbf{3 . 1 8}$ & 4.45 & 6 \\
\hline MP & -2.84 & -0.32 & $\mathbf{4 . 5 7}$ & 3.78 & 15 \\
\hline EMP & -5.72 & -2.6 & $\mathbf{6 . 3}$ & 6.86 & 15 \\
\hline PTB & 0.56 & 2.34 & $\mathbf{5 . 8 4}$ & 6.36 & 12 \\
\hline
\end{tabular}

TABLE III

PREDISTORTION EVM IMPROVEMENT FOR DIFFERENT OPTICAL INPUT LEVELS AT $P_{r e f}=-14$ DBM

- In Fig. 4.a, it is seen that a MP parametric complexity of $\left(N_{3}=3\right.$ and $\left.M_{1}=5\right)$ provides the best performance at a limited parametric complexity (10 coefficients). One should remember that the main region of interest concerns high power 
values where the SOA operates close to the identification power $\left(P_{\text {ref }}=-14 \mathrm{dBm}\right)$. Hence when $P_{\text {in }}<-20 \mathrm{dBm}$, only linear predistorters are providing a gain in EVM.

- In Fig. 4.b, EMP plots are similar to MP ones. A third order EMP predistorter with a memory depth of 5 provides the best results when $P_{i n}$ is close to the identification optical power $P_{r e f}=14 \mathrm{dBm}$. When the operating point is moving towards the linear region $\left(P_{i n} ; 18 \mathrm{dBm}\right)$ it can be observed that all the predistorters are degrading the CO-OFDM transmitter performance. Fig. 4.b

- In Fig. 4.c PTB predistorters present performances comparable to MP ones when the component exhibits nonlinear effects (order $>1$ ). However best results are obtained when the dynamics is linear. It is hence seen that the two-box structure with a constrained static bloc and linear dynamic (FIR) is providing improvement for a wide range of optical input powers and is therefore more robust to $P_{\text {in }}$ variations.

The overall performance of STATIC, MP, EMP and PTB predistortion are compared in Fig. 4.d and Tab. III. EMP predistortion presents the best EVM gain at high power, slightly better than PTB predistortion. EMP predistortion is however not very robust to decreasing $P_{i n}$ values contrary to PTB predistortion which is providing improvement for input power as low as $P_{i n}=-26$ $\mathrm{dBm}$. As for the two other predistorters they are inferior in performance. MP predistortion is however more efficient than constrained STATIC predistortion for $P_{i n}$ ranging from $-22 \mathrm{dBm}$ to $-12 \mathrm{dBm}$.
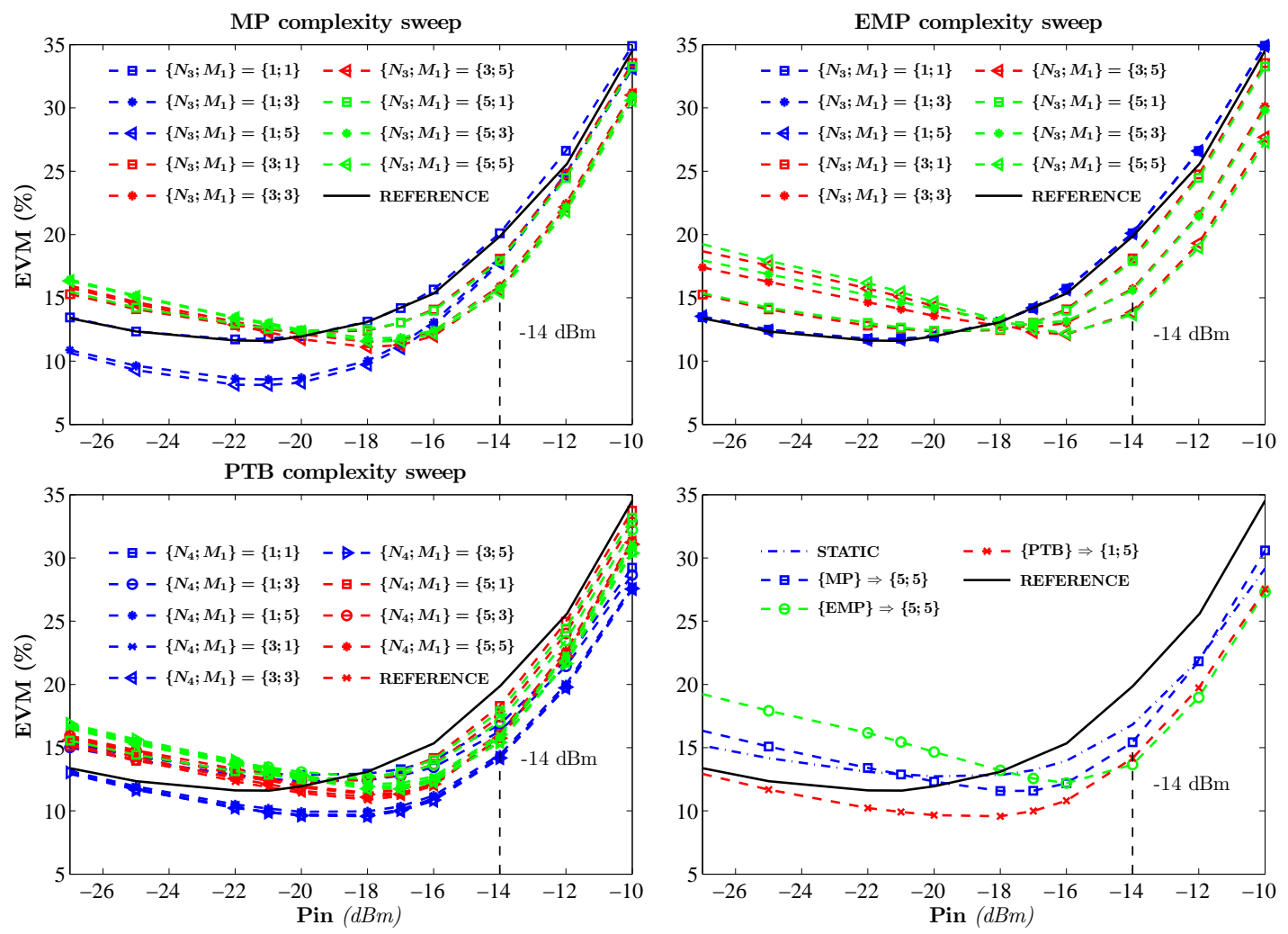

Fig. 4. Predistortion parametric complexity sweep for an identification power at $\left.P_{r e f}=-14 \mathrm{dBm}, \mathrm{a}\right) \mathrm{MP}$, b) EMP, c) PTB and d) comparison plot

2) Optical input power influence: The previous results are relative to an identification at $P_{\text {ref }}=-14 \mathrm{dBm}$. We now present validation results for $P_{r e f}=-18 \mathrm{dBm}$ (low power) and $P_{r e f}=-11 \mathrm{dBm}$ (high power). Orders and memory depth are kept the same (Tab. III). Predistortion performance is compared in Fig. 5. The basic trend is that increasing identification input power $\left(P_{r e f}\right)$ will allow for more predistortion gain in high power and degraded EVM in low power.

- For $P_{\text {ref }}=-18 \mathrm{dBm}$, close to the linear regime of the SOA (Fig. 5.a), MP and PTB predistortion have very similar behavior. EMP presents the same performance in high power and a poor performance in low power. The less efficient structure is the STATIC predistorter.

- For $P_{r e f}=-11 \mathrm{dBm}$, in a very saturated operating point (Fig. 5.b), as for $P_{i n}=-14 \mathrm{dBm}$, EMP and PTB predistorters have close performance in high power. However EMP scheme is very sensitive to the decrease in $P_{i n}$. For instance at $P_{i n}=-16 \mathrm{dBm}$, EMP predistortion induces a loss in EVM of 3\% while PTB predistortion still provides an EVM gain of $2 \%$. MP predistortion is very unefficient, having a performance inferior to the STATIC predistortion all over presented optical input power range.

In Fig. 5.c and Fig. 5.d PAPR is evaluated at the output of various predistorters, for $P_{\text {ref }}=-18 \mathrm{dBm}$ and $P_{\text {ref }}=-11$ $\mathrm{dBm}$. At low power the PTB design induces the highest crest factor $(11.7 \mathrm{~dB})$ followed by EMP, MP and STATIC approaches 

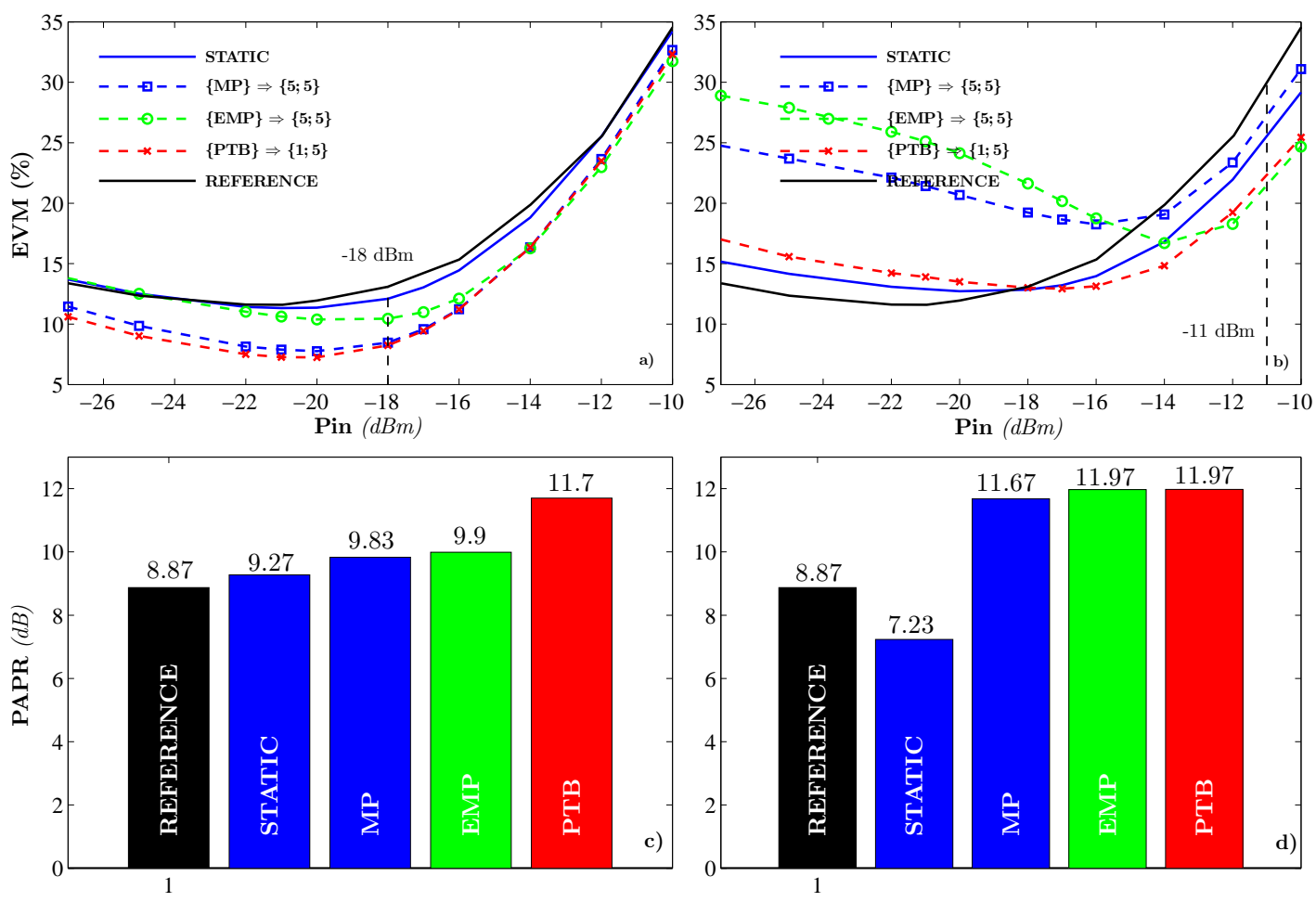

Fig. 5. Predistortion performance at a) $P_{r e f}=-18 \mathrm{dBm}$, b) $P_{r e f}=-11 \mathrm{dBm}$ and Predistortion PAPR c) $P_{r e f}=-18 \mathrm{dBm}$ and d) $P_{r e f}=-11 \mathrm{dBm}$

with a PAPR of $9.9 \mathrm{~dB}, 9.83 \mathrm{~dB}$, and $9.27 \mathrm{~dB}$, respectively, while the original OFDM signal exhibits a PAPR of $8.87 \mathrm{~dB}$. For $P_{r e f}=-11 \mathrm{dBm}$ multiple remarks can be derived. STATIC predistortion actually induces a PAPR reduction. While reference electrical OFDM PAPR is unchanged $(8.87 \mathrm{~dB})$, the static predistorter leads to a PAPR of $7.23 \mathrm{~dB}$. In fact due to the high power identification level, the non-constrained memoryless predistorter is less efficient (Fig. 3.b). Constrained static identification is yielding optimal coefficients that induces a PAPR reduction. It has also to be noted that MP and EMP predistortion are very sensitive to saturated operating point identification. They respectively present 11.97 and $11.67 \mathrm{~dB}$ of PAPR while PTB PAPR is at $11.97 \mathrm{~dB}$. However high order polynomial predistorters identified at a very saturated operating point induce low performance when operating at a input power far inferior to the identification reference (i.e $P_{r e f}=-11$ $\mathrm{dBm}$ ). Impairments precompensation is provided when the SOA exhibits linear behavior. Comparatively, the PTB design which is providing good performance without the need of high polynomial orders has a significant better performance at low power.

3) BER performance: The EVM criterion has the advantage that it can be easily evaluated whatever the operating point is, but the Bit Error Rate (BER) is more representative of the system performance from a practical point of view. Estimating the BER from the EVM in presence of strong nonlinear effects may lead to erroneous conclusions, so we conducted Monte Carlo simulations so as to evaluate the BER. Due to the relatively low EVM observed over the whole operating range, a large computational burden has been required for this evaluation. For this reason we considered only the case where the predistorters are identified at $P_{r e f}=-11 \mathrm{dBm}$, yielding EVM values above $20 \%$. The results are depicted in Fig. 6 . It can be seen that the EMP design offers the best performance in the vicinity of $P_{r e f}$, with a clear advantage over the PTB design, but keeping in mind that a crossing between the BER curves should occur around $-12 \mathrm{dBm}$ (which does not appear on the plot due to the difficulty to evaluate BERs of the order of $10^{-6}$ ). The sensitivity of the EMP predistorter to optical power, with respect to $P_{\text {ref }}$ can be seen at $P_{i n}=-8 \mathrm{dBm}$ where a close performance is obtained for the PTB and EMP designs. Another observation is that the proposed Static design offers a close BER performance to the PTB when operating at high power, as a result of PAPR reduction.

4) DAC/ADC resolution influence: The 12 bits resolution considered so far for the DAC/ADC may not be realistic in many practical applications. We subsequently examine the inuence of an increased quantization step using 4 bits, which translates into a significant change in signal dynamics and may lead to a different behavior for the considered predistorters. We focus on the case of an identification at $-11 \mathrm{dBm}$, where the nonlinear effects are the more significant, and keeping the same values as before for the predistorters order and memory length. The results are depicted in Fig. 7. Again, the MP scheme appears to be the less effective approach, with very few EVM improvement at $P_{r e f}$ and a high sensitivity to optical power variation. The EMP predistorter offers the better performance in the vicinity of the reference power, with a slightly more pronounced 


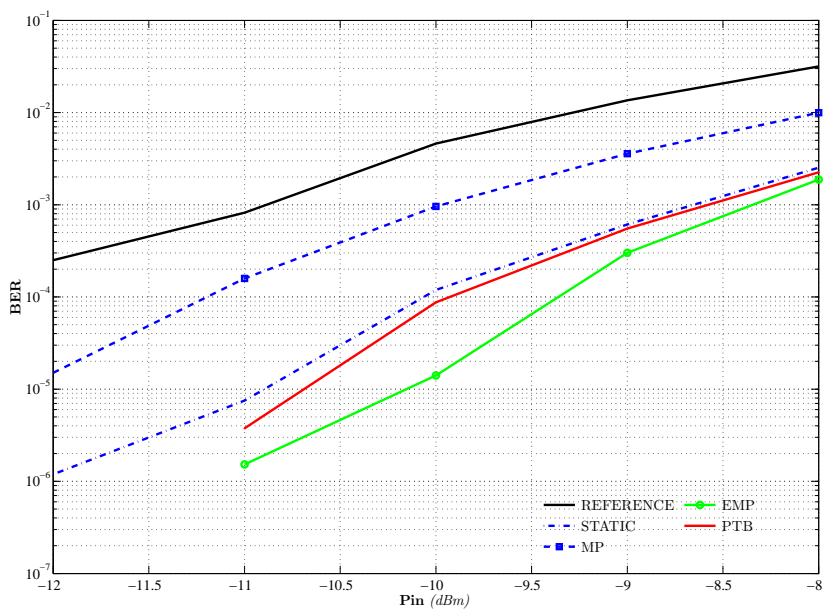

Fig. 6. BER against SOA input power for an identification at $P_{\text {ref }}=-11 \mathrm{dBm}$

advantage over the PTB scheme, but once again the EMP method is not robust when the operating point deviates from the identification point. It can be seen that the PTB technique gives the best tradeoff as previsously observed for a finer DAC/ADC resolution. The constrained static design could also be considered as a pertinent tradeoff if a very low complexity is targeted.

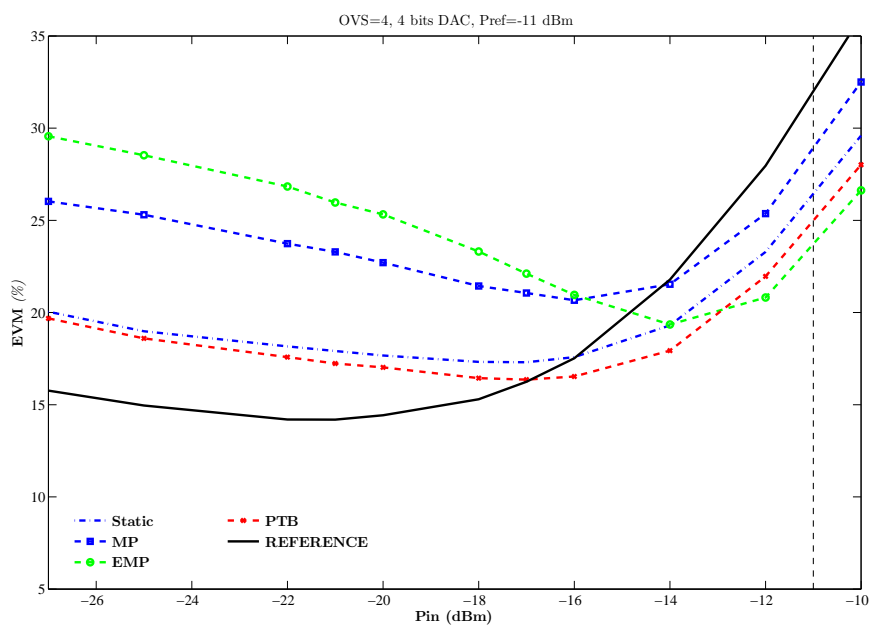

Fig. 7. Predistortion performance with a DAC/ADC resolution of 4 bits, for an identification at $P_{r e f}=-11 \mathrm{dBm}$

\section{RoBUSTNESS ANALYSIS}

Robustness analysis is presented throughout this section. The analysis will focus on five different cases. First we will study robustness with respect to IQ modulator peak-to-peak Voltage $\left(V_{p p}\right)$. Then SOA biasing current $\left(I_{\text {bias }}\right)$ and laser wavelength $(\lambda)$ variation impact are evaluated. The impact of the number of subcarriers on predistortion performance is also assessed. Finally the default SOA INPHENIX-IPSAD1501 model is tweaked, through its Henry factor $\left(\alpha_{H}\right)$ to exhibit a more pronounced nonlinear behavior. Predistortion performance is then studied in this configuration. By default, validation simulations are performed with $2^{13} 4$-QAM symbols while models were identified with $2^{15} 4$-QAM symbols.

\section{A. IQ modulator Voltage sweep}

Robustness against IQ modulator Peak-to-Peak Voltage $\left(V_{p p}\right)$ will be studied for two different test cases: At low power where the SOA exhibits a quasi-linear behavior and at high power where the device is operating in saturation mode. We proceed as follows; default $V_{p p}$ voltage is first set at 8 Volts, the voltage used so far. Then optical attenuation is determined to meet two input power settings: $-21 \mathrm{dBm}$ (low power case) and $-13 \mathrm{dBm}$ (high power case). Once determined, attenuation values are left unchanged. Robustness analysis will then consist in sweeping $V_{p p}$ from 1 Volts to 15 Volts and in assessing EVM evolution. In Fig. 8.a, input power $P_{i n}$ against $V_{p p}$ is plotted. A nonlinear dependence is observed. In Fig. 8.b, EVM against 
$V_{p p}$ is presented for the first test case $\left(-21 \mathrm{dBm}\right.$ initial setting). For decreasing $V_{p p}$ values under 3 Volts, EVM is rapidly growing up due to low optical input power $\left(P_{i n} \approx-35 \mathrm{dBm}\right)$ and a significant impact of ASE noise on the transmission quality (low OSNR). For high $V_{p p}$ values, as SOA is operating close to its saturation point, EVM is also increasing due to nonlinear impairments, the rate is however slower. In Fig. 8.b, identified STATIC, MP, EMP and PTB predistorters are improving system performance when $V_{p p}>3 \mathrm{~V}$ corresponding to $P_{i n}=-31 \mathrm{dBm}$. MP and PTB predistortion offer the best EVM improvement and have close behaviors. For $V_{p p}$ close to 14 Volts, up to $5 \%$ of EVM gain is obtained. A value wich is consistent with EVM improvement for optical input power $P_{i n}$ close to $-20 \mathrm{dBm}$ (Fig. 8.a and Fig. 5.a). In fact controlling $V_{p p}$ is an indirect way for setting input optical power $P_{i n}$ as shown in Fig. 8.a. EVM against $V_{p p}$ is thus directly related to EVM against input power, $P_{i n}$ being either set through an attenuator or indirectly through $V_{p p}$. This is verified with the second test case. For this test case ASE effect (low $V_{p p}$ values) and saturation impairments (high $V_{p p}$ values) are still present leading to EVM loss. Saturation impairments are however more pronounced and EVM is rapidly increasing for high $V_{p p}$ values. This is due to the initial -13 $\mathrm{dBm}$ power setting. For the very saturated region (high $V_{p p}$ ) EMP predistortion is providing the best improvement up to $11.6 \%$ for $V_{p p}=14$ Volts corresponding to $P_{i n}$ close to $-8.5 \mathrm{dBm}$. It is followed by PTB predistortion (8.4\%), constrained static predistortion (6.67\%), and finally MP (5.52\%) (Fig. 8.c and Tab. IV). For $V_{p p}=2$ Volts and $P_{i n} \approx-26 \mathrm{dBm}$ PTB (-2.41\%) and Static $(-3.38 \%)$ predistortion present less EVM degradation. As it could be expected refering to Fig. 5.b. EMP is presenting the worst performance with an EVM loss of $-14.44 \%$ (Tab. IV).

\begin{tabular}{|c|c|c|c|c|}
\hline$V_{p p}($ Volts $)$ & 2 & 6 & 10 & 14 \\
\hline$P_{i n}(\mathrm{dBm})$ & -26.05 & -16 & -11.21 & -8.52 \\
\hline \hline STATIC & -3.38 & 1.8 & 6.14 & 6.67 \\
\hline MP & -10.79 & -1.74 & 4.04 & 5.52 \\
\hline EMP & -14.44 & -1 & 9.51 & 11.6 \\
\hline PTB & -2.41 & 3.57 & 7.61 & 8.4 \\
\hline
\end{tabular}

TABLE IV

PREDISTORTION EVM IMPROVEMENT FOR DIFFERENT VALUES OF PEAK-TO-PEAK VOLTAGE
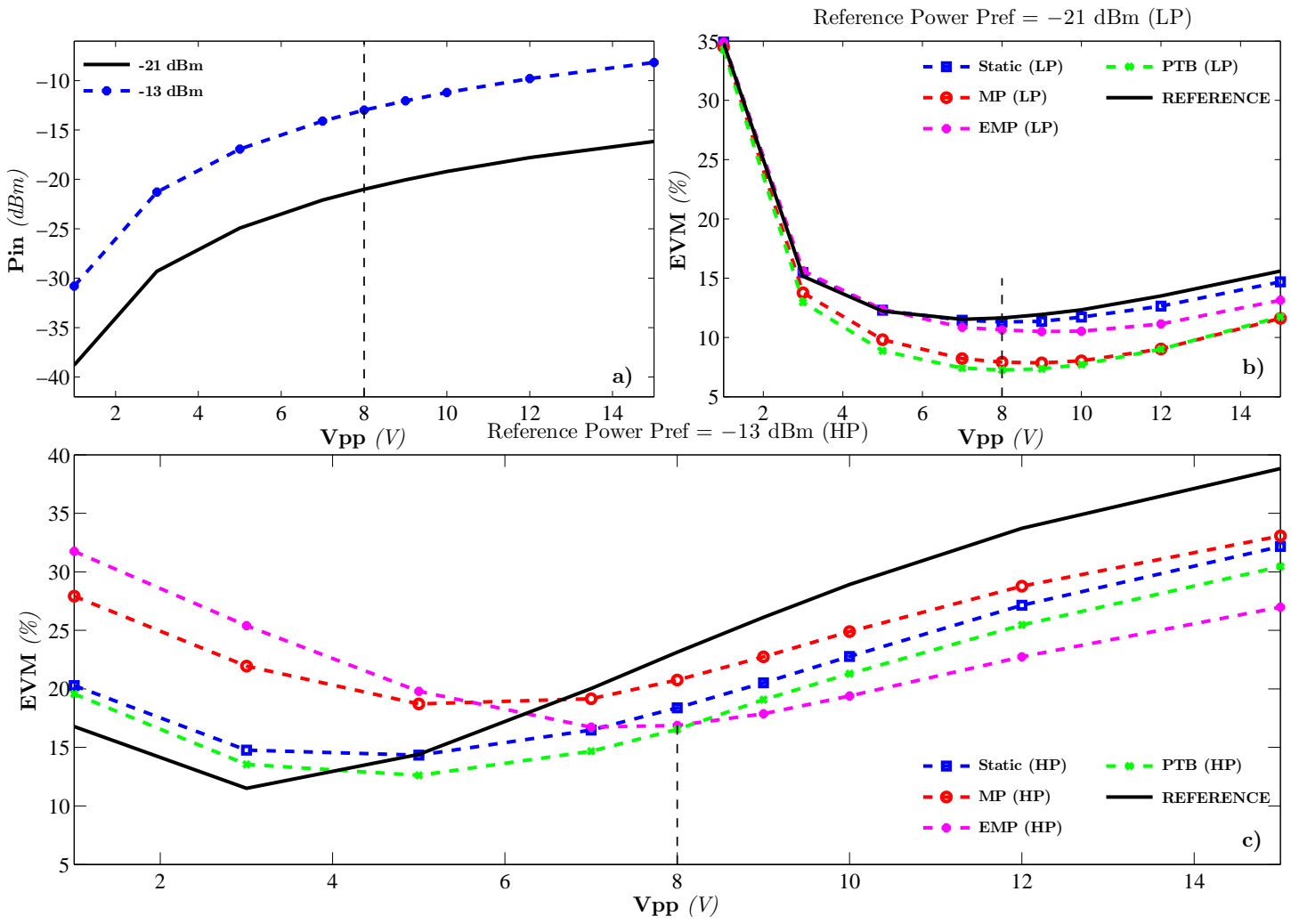

Fig. 8. a) Input optical power against Peak-to-Peak Voltage, b) EVM against Peak-to-Peak Voltage for a -21 dBm input power, c) EVM against Peak-to-Peak Voltage for a $-13 \mathrm{dBm}$ input power 


\section{B. Biasing current influence}

So far simulations were carried out for a fixed $150 \mathrm{~mA}$ injection current $\left(I_{\text {bias }}\right)$ setting. We now proceed to biasing current sweep from $117 \mathrm{~mA}$ to $220 \mathrm{~mA}$. Two initial power levels are considered, $-21 \mathrm{dBm}$ (low power case) and $-13 \mathrm{dBm}$ (high power case). As presented in Fig. 9.a, the direct effect of injection current variation is a change in SOA gain. In Fig. 9.a gain evolution with respect to both input power $\left(P_{i n}\right)$ and bias current $\left(I_{b i a s}\right)$ is plotted. SOA gain is increasing with the biasing current. In Fig. 9.b we also observe that higher biasing current results in degraded EVM performance. We compared predistortion performance for two reference levels, $-21 \mathrm{dBm}$ and $-13 \mathrm{dBm}$ (Fig. 9.c). At $-21 \mathrm{dBm}$ reference EVM (11.6\%) and predistortion EVM is quasi-constant (less than 1\% variation) with respect to biasing current over the range [120,220] mA. In this case PTB is providing the best performance followed by MP, EMP and static predistorters, with an EVM improvement of 4.6, 4, 2, $0.5 \%$, respectively. At $-13 \mathrm{dBm}$ (Fig. 9.c and Tab. V), EMP predistortion is providing best performance at high power and the worst at low power; EVM improvement is up to $11.13 \%$ at $220 \mathrm{~mA}$. PTB, static and MP EVM gains are respectively at $9.17,7.39$ and $4.74 \%$ for the same biasing current value. One should also notice that for the reference $I_{\text {bias }}=150 \mathrm{~mA}$, PTB and EMP have equivalent performance (17\% of EVM). EMP is providing up to $2 \%$ superior performance at $I_{\text {bias }}=220 \mathrm{~mA}$. In Fig. 9.d, we plotted EVM against output power $P_{\text {out }}$ instead of $P_{\text {in }}$ or $I_{\text {bias }}$. A higher output power is providing a longer transmission reach. We are now comparing EVM against $P_{\text {out }}$ variations, first by controlling optical attenuation just before the SOA and then through directly increasing $I_{\text {bias }}$. EVM against $P_{\text {out }}$ without predistortion and with EMP predistortion is plotted for both approaches. It can be clearly observed that a better predistortion performance may be achieved if the reach is extended by increasing the bias current. For instance at a given optical output power $P_{\text {out }}=4.5 \mathrm{dBm}$ and a $220 \mathrm{~mA} I_{\text {bias }}$ setting, EMP predistortion is providing up to $14 \%$ of EVM improvement; while for the same output power and a bias current lowered at $150 \mathrm{~mA}$ EMP improvement decreases to 11

\begin{tabular}{|c|c|c|c|c|}
\hline Biasing current $(\mathrm{mA})$ & 120 & 160 & 200 & 220 \\
\hline \hline STATIC & 0.11 & 5.56 & 6.9 & 7.39 \\
\hline MP & -3.5 & 3.12 & 4.31 & 4.74 \\
\hline EMP & -4.53 & 7.94 & 10.38 & 11.13 \\
\hline PTB & 1.86 & 7.33 & 8.66 & 9.17 \\
\hline
\end{tabular}

TABLE V

PREDISTORTION EVM IMPROVEMENT FOR VARYING BIASING CURRENT

\section{Wavelength shift influence}

We also study robustness with respect to laser wavelength $\lambda$ which is swept from $1500 \mathrm{~nm}$ to $1560 \mathrm{~nm}$ (reference at 1540 $\mathrm{nm}$ ). SOA gain evolution is presented in Fig. 10.a, attaining a peak around $1520 \mathrm{~nm}$. EVM against $P_{\text {in }}$ curves for different wavelengths are then plotted in Fig. 10.b. At $-21 \mathrm{dBm}$, reference EVM is close to $12 \%$ and is stable with respect to $\lambda$ variations. PTB and MP predistorters are offering the highest performance around 5\% of stable EVM improvement for each predistorter. At higher power $(-13 \mathrm{dBm})$, EMP predistortion offers the best performance for $\lambda<1540 \mathrm{~nm}$. For $\lambda=1520 \mathrm{~nm}$ (Tab. VI), an improvement of $9.21 \%$ is observed for the EMP predistorter; PTB, STATIC and MP designs offer an improvement of $8.04 \%, 5.88 \%$ and $4.43 \%$, respectively. As the SOA operates close to its saturation gain at $1520 \mathrm{~nm}$, impairments are more pronounced and EMP offers the best performance. It is also noticed that when $\lambda$ is close to $1560 \mathrm{~nm}$, EMP and MP predistorters perform worst, inducing respectively an EVM loss of $-3.29 \%$ and $-3.43 \%$. This is due to the device's lower gain. The SOA is getting close to a linear operating point and (EMP, MP) identified at $P_{r e f}=-11 \mathrm{dBm}$ are less efficient. The PTB still provides a $2.06 \%$ EVM improvement while constrained static predistortion offers providing around $1 \%$ of improvement.

\begin{tabular}{|c|c|c|c|c|}
\hline$\lambda(\mathrm{nm})$ & 1500 & 1520 & 1540 & 1560 \\
\hline \hline STATIC & 4.87 & 5.88 & 4.73 & 0.98 \\
\hline MP & 3.68 & 4.43 & 2.34 & -3.43 \\
\hline EMP & 8.23 & 9.21 & 6.61 & -3.29 \\
\hline PTB & 6.44 & 8.04 & 6.24 & 2.06 \\
\hline
\end{tabular}

TABLE VI

PREDISTORTION EVM IMPROVEMENT FOR VARYING INPUT WAVELENGTH; $P_{r e f}=-13$ DBM

\section{Number of subcarriers sweep}

We now consider a varying number of subcarriers $\left(N_{s c}\right)$ from 64 to 2048 . To be consistent with the digital predistorter's sampling period we accordingly sweep symbol time $T_{u}$ from $12.8 \mathrm{~ns}$ to $409.6 \mathrm{~ns}$ (initial $T_{u}=25.6 \mathrm{~ns}$ is taken as reference). Bandwidth is thus constant. Simulations are now carried out with a sequence of $2^{15} 4$-QAM symbols. Results are shown in (Fig. 11). for low $(-21 \mathrm{dBm})$ and high power $(-13 \mathrm{dBm})$. We observe, for both cases, a slight deviation in EVM when number of subcarriers is varying (Fig. 11.a and Fig. 11.b). As presented in previous paragraphs, predistortion is more sensitive to $P_{\text {out }}$ 

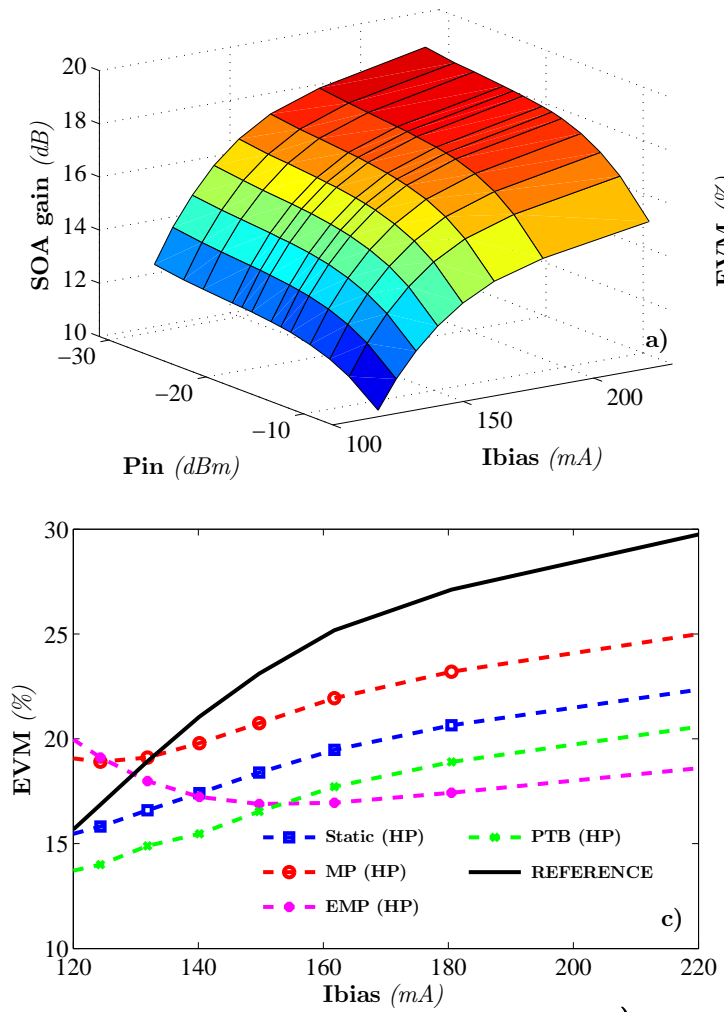
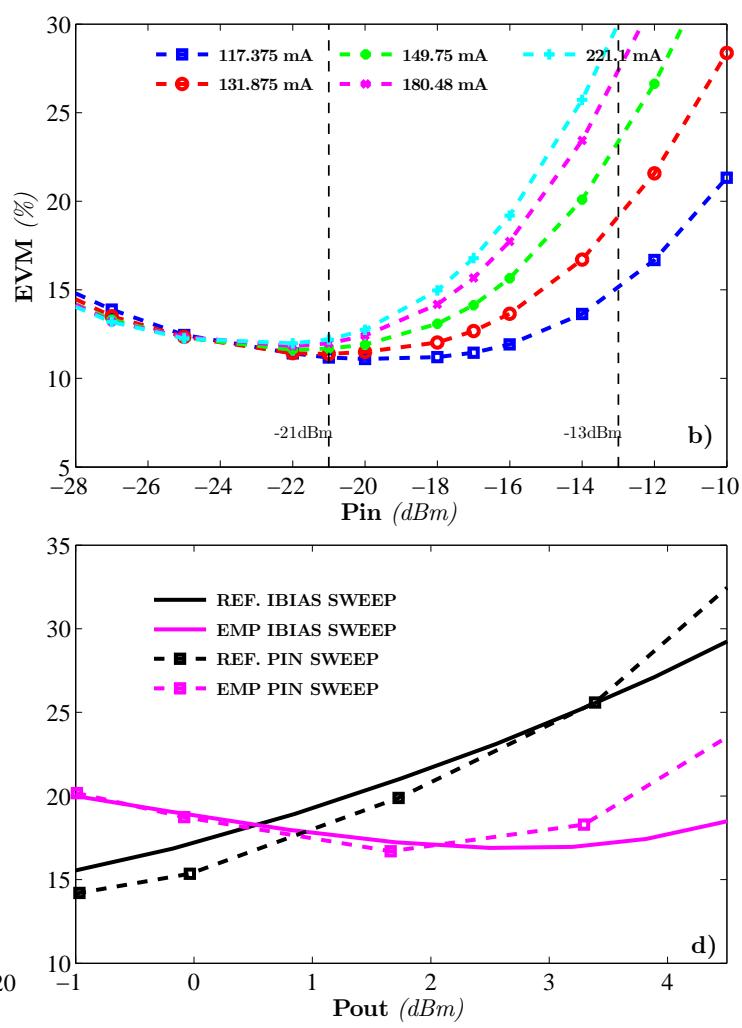

Fig. 9. a) SOA gain with respect to injected power and biasing current static variations, b) EVM against injected power for different biasing current values c) EVM against biasing current at a fixed $P_{r e f}$ at $-13 \mathrm{dBm}$, d) EVM against Output power by varying input power directly through attenuation or via biasing current

variations either via $P_{i n}, V_{p p}$, biasing current or input wavelength $\lambda$ particularly for saturation point identification. We rather observe a robust behavior with respect to $N_{s c}$ variation either at low or high power.

\section{E. Influence of phase-amplitude coupling}

We assess predistortion performance and robustness in the case of a SOA exhibiting much stronger phase-amplitude coupling. To that end SOA Henry factor $\alpha_{H}$ which was previously set at 3.3 is now set at 5 . A new identification has been carried out for static, EMP and PTB predistortion with $P_{r e f}=-14 \mathrm{dBm}$. MP predistortion providing lower performance in case of strong nonlinearities is not presented. In Fig. 12.a the general plot representing EVM with respect to input power $P_{i n}$ and $\alpha_{H}$ is plotted. Greater EVM values are obtained when $\alpha_{H}$ is increasing. In Fig. 12.b a comparison of EVM against input power, for the two distinct values of the Henry factor, is also plotted. In Tab. VII we compare the second order static predistorter optimal ordinate $y_{c}^{*}$ for the two values of Henry factor. As increasing $\alpha_{H}$ generates higher nonlinearities the optimal ordinate $y_{c}^{*}$ is decreasing (see paragraph IV-A).

\begin{tabular}{|c|c|c|c|}
\hline$P_{\text {ref }}$ & $-18 \mathrm{dBm}$ & $-14 \mathrm{dBm}$ & $-11 \mathrm{dBm}$ \\
\hline$\alpha_{H}=3.3$ & 2.1 & 1.75 & 1.62 \\
\hline$\alpha_{H}=5$ & 1.94 & 1.36 & 1.01 \\
\hline
\end{tabular}

TABLE VII

OPTIMAL CONSTRAINED STATIC PREDISTORTER ORDINATE $y_{c}^{*}$ FOR $\alpha_{H}=3.3$ AND $\alpha_{H}=5$

Then EMP predistortion and PTB predistortion are compared in Fig. 12c and Table VIII for $\alpha_{H}=3.3$ and $\alpha_{H}=5$ at $P_{r e f}=-14 \mathrm{dBm}$. EMP and PTB predistortion present a close improvement at high power; for $P_{i n}=-12 \mathrm{dBm}$ they respectively offer an improvement of $5.86 \%$ and $6.62 \%$ in EVM. In the case of $\alpha_{H}=5$, EMP and PTB predistortion are respectively providing $9.71 \%$ and $9.89 \%$ in EVM improvement for the same input level. Still for $P_{i n}=-12 \mathrm{dBm}$, reference EVM is at $25 \%$ and $35 \%$ respectively for $\alpha_{H}=3.3$ and $\alpha_{H}=5$.

\section{CONCLUSION}

Various digital baseband predistorters have been investigated in this paper for improving the EVM performance of an SOAbased 17 Gbps CO-OFDM transmitter, while considering the criterion of robustness against system parametric variations. A 

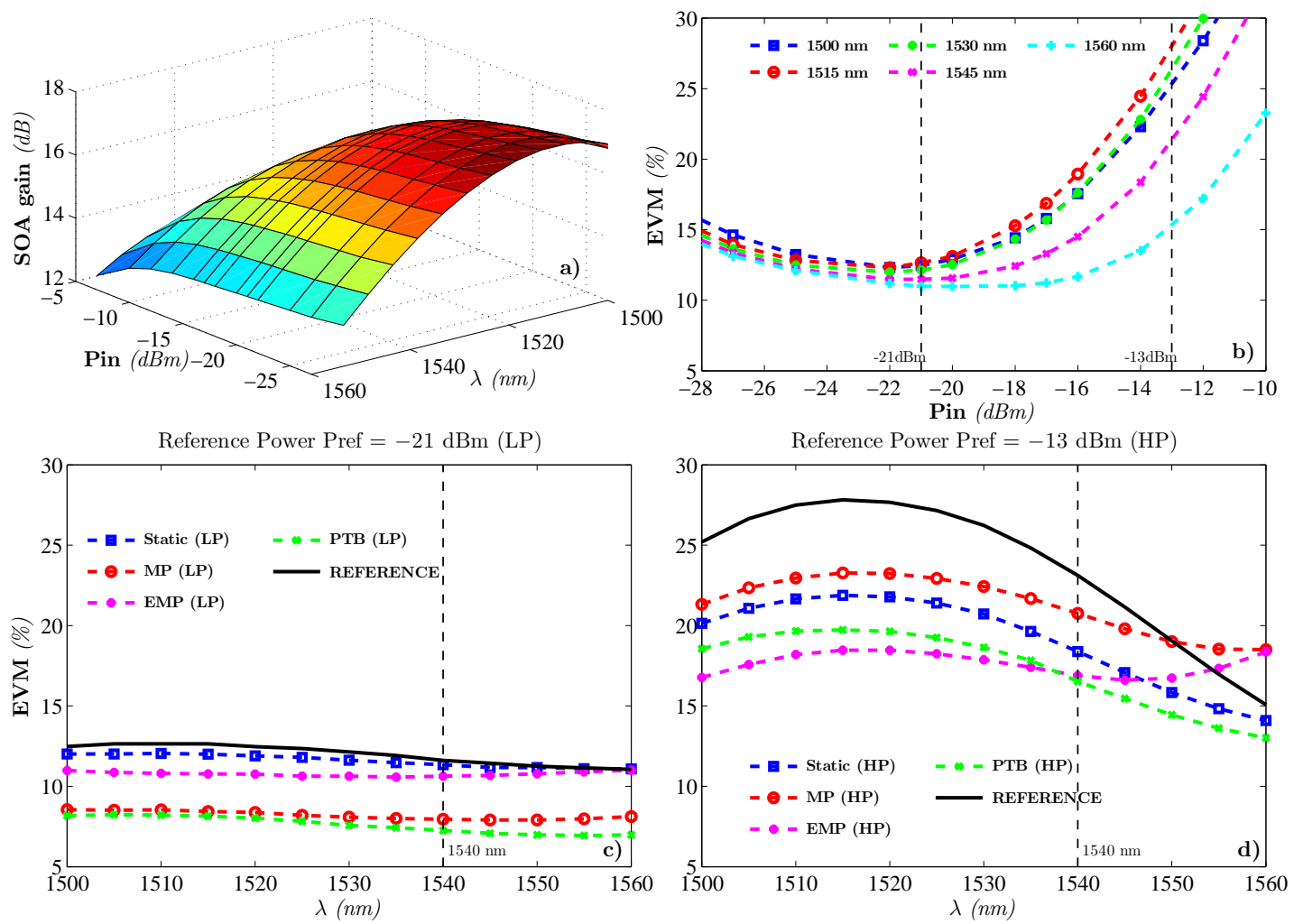

Fig. 10. a) SOA gain with respect to injected power and input wavelength variations, b) EVM against injected power for different input wavelengths c) EVM against input wavelength at a fixed $P_{i n}$ at $\left.-21 \mathrm{dBm}, \mathrm{d}\right)$ EVM against input wavelength at a fixed $P_{i n}$ at $-13 \mathrm{dBm}$.
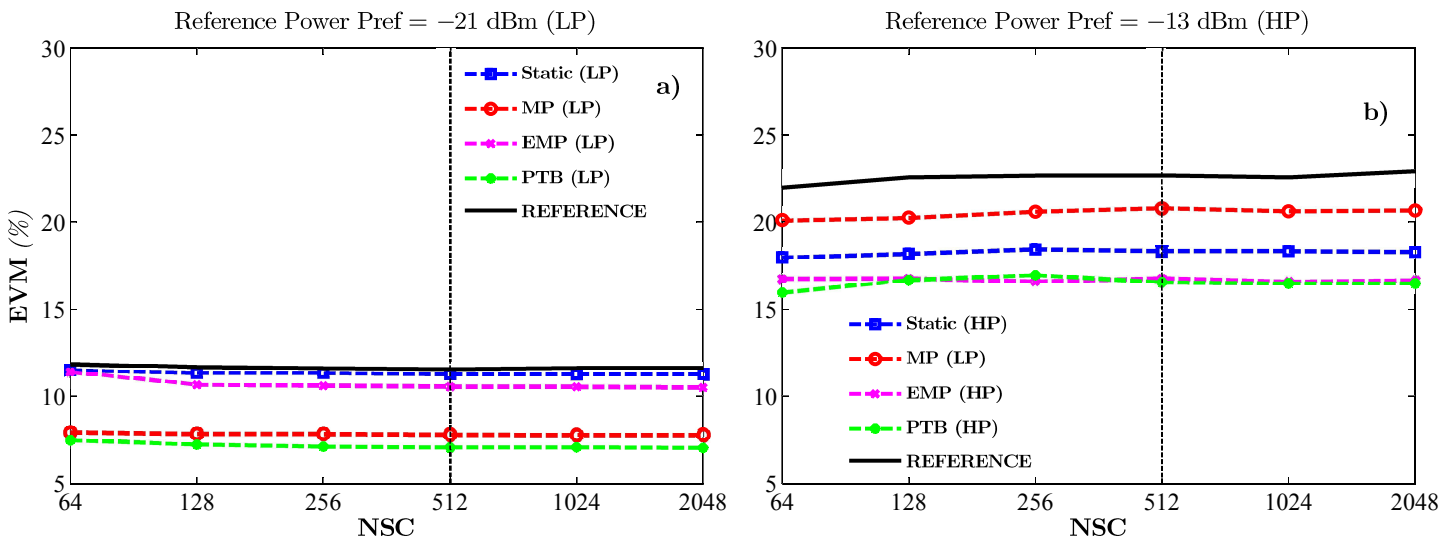

Fig. 11. a) EVM against Number of subcarriers at $P_{\text {in }}=-21 \mathrm{dBm}$, b) EVM against Number of subcarriers at $P_{\text {in }}=-13 \mathrm{dBm}$

special focus is given to a parallel two-box (PTB) behavioral model, combining a static nonlinear function with a memory polynomial (MP) model. A simple design of the static function has been first proposed for jointly achieving linearization and reduction of the peak amplitudes. Then, the study of the parametric complexity of the different predistorters (static, MP, EMP, PTB) revealed that the PTB scheme with constrained static and linear dynamics (FIR) is providing the best performance, with an EVM improvement over a wide range of optical input power with a single identification at $-14 \mathrm{dBm}$. Furthermore, it is shown that predistortion robustness against optical input power variation is strongly dependent on the operating point chosen for identification. Generally speaking, the polynomial predistorters studied throughout the paper are sensitive to output optical power uctuations, either directly, or through parameters such as IQ modulator peak-to-peak voltage, SOA biasing current or even input wavelength. Overall, the PTB structure offers the best compromise. It is a low-complexity design capable of lowering EVM over a wide range of transmitter parameter values. 

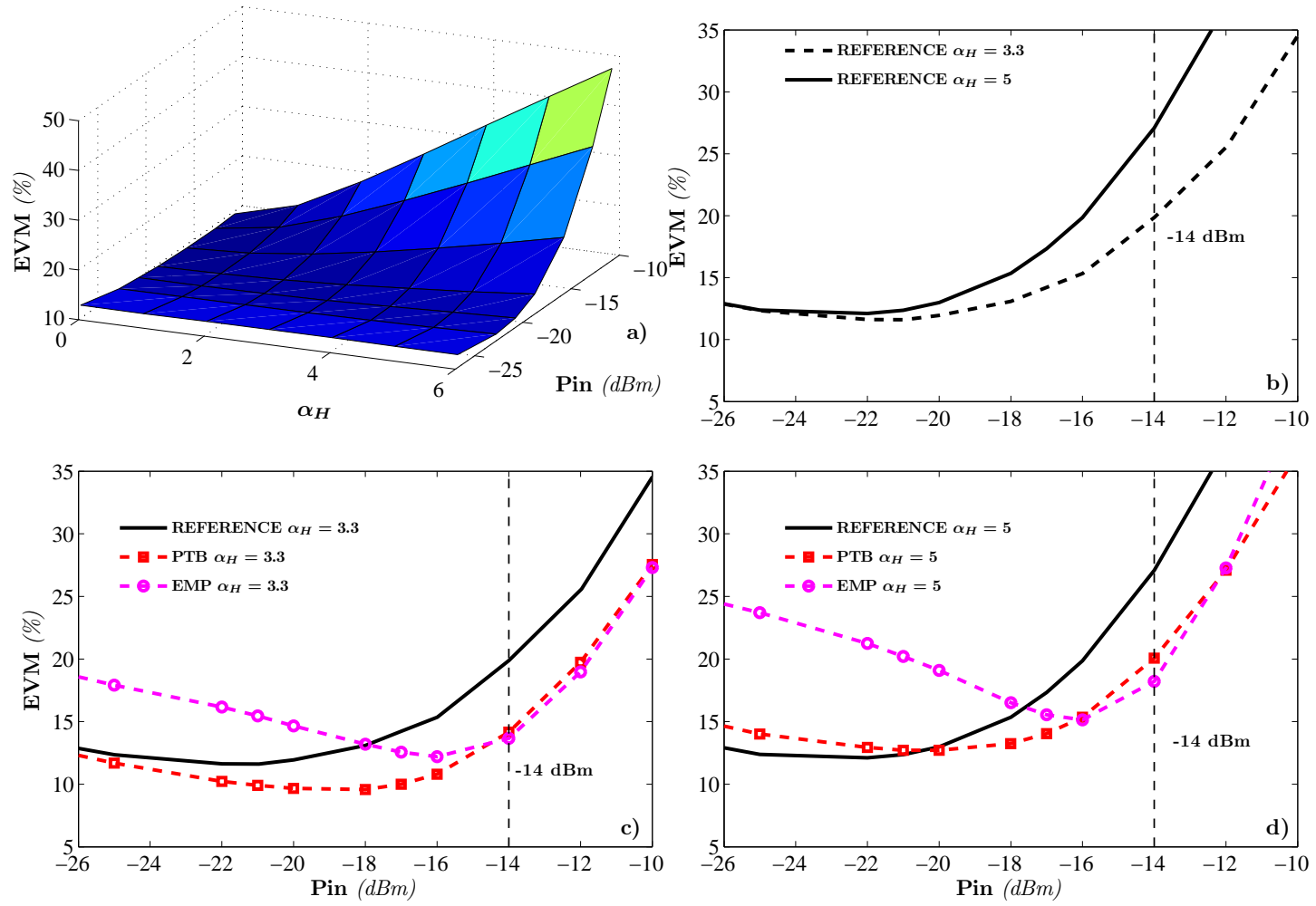

Fig. 12. a) EVM against injected power and Henry factor, b) EVM against injected power for $\alpha_{H}=3.3$ and $\alpha_{H}=3.3$, c) predistortion EVM performance for $\alpha_{H}=3.3$, d) predistortion EVM performance for $\alpha_{H}=5$.

\begin{tabular}{|c|c|c|c|c|}
\hline Pin $(\mathrm{dBm})$ & -25 & -20 & -16 & -12 \\
\hline \hline EMP, $\alpha_{H}=5$ & -11.31 & -6.1 & 4.72 & 9.71 \\
\hline PTB, $\alpha_{H}=5$ & -1.63 & 0.27 & 4.45 & 9.89 \\
\hline EMP, $\alpha_{H}=3.3$ & -5.57 & -2.7 & 3.15 & 6.62 \\
\hline PTB, $\alpha_{H}=3.3$ & 0.66 & 2.28 & 4.54 & 5.86 \\
\hline
\end{tabular}

TABLE VIII

EMP AND PTB PREDISTORTION EVM IMPROVEMENT FOR $\alpha_{H}=3.3$ AND $\alpha_{H}=5$ AT $P_{r e f}=-14$ DBM

\section{REFERENCES}

[1] W. Shieh and I. B. Djordjevic, OFDM for Optical Communications, (Elsevier/Academic Press, 2009).

[2] A. J. Lowery, L. B. Du, "Optical orthogonal division multiplexing for long haul optical communications: A review of the first five years", Optical Fiber Technology 17 (2011) 421-S438.

[3] N. Cvijetic, "OFDM for Next-Generation Optical Access Networks", J. Lightw. Technol., vol. 30, no. 4, feb. 2012.

[4] H. Ochiai and H. Imai, "On the Distribution of the Peak-to-Average Power Ratio in OFDM Signals", IEEE Trans. Commun., vol. 49, no. 2, pp. 282-289, Feb. 2001.

[5] Y. Rahmatallah and S. Mohan, "Peak-to-average power ratio reduction in OFDM systems: A survey and taxonomy", IEEE Communications Surveys and Tutorials, vol. 15, no. 4, 2013

[6] B. Goebel, S. Hellerbrand, N. Haufe and N. Hanik, "PAPR reduction techniques for coherent optical OFDM transmission", in Proc. ICTON 2009, Jul. 2009.

[7] Amiralizadeh, S., Nguyen, A. T., and Rusch, L. A., "Modeling and compensation of transmitter nonlinearity in coherent optical OFDM", Optics express, 23(20), 2015

[8] H. Khaleghi, P. Morel, A. Sharaiha, and T. Rampone, Experimental Validation of Numerical Simulations and Performance Analysis of a Coherent Optical-OFDM Transmission System Employing a Semiconductor Optical Amplifier, IEEE J. Lightwave Technol., vol. 31, no. 1, pp. 161-170, January 2013.

[9] S. Bejan, S. Azou, P. Morel, C. Diouf, M. Telescu, N. Tanguy, A. Sharaiha, "A Joint Linearization/Companding Approach for Improving a CO-OFDM Transmitter", IEEE Photon. Technol. Lett., vol. 27, no. 20, pp. 2162-2165, 2015.

[10] M. J. Connelly, Semiconductor Optical Amplifiers. Boston, MA:Kluwer, 2002.

[11] H. Schmuck et al., "Demonstration of an SOA-assisted open metro-access infrastructure for heterogeneous services", Optics Express, vol. 22, Issue 1, 2014.

[12] C. Tai, S.-L. Tzeng, H.-C. Chang, and W. I. Way, "Reduction of nonlinear distortion in mqw semiconductor optical amplifier using light injection and its application in multichannel m-qam signal transmission systems", IEEE Photon. Technol. Lett. , vol. 10, pp. 609-611, Apr. 1998.

[13] F. Tabatabai, H. S. Al-Raweshidy, "Feed-Forward Linearization Technique for Reducing Non-Linearity in Semiconductor Optical Amplifier", J. Lightw. Technol., vol.25, No. 9, pp.2667-2674, Sep. 2007. 
[14] X. Zhang, R. Zhu, D. Shen, and T Liu, "Linearization Technologies for Broadband Radio-Over-Fiber Transmission Systems," Photonics, vol. 1, no.4, pp. 455-472, 2014

[15] F. M. Ghannouchi, O. Hammi, and M. Helaoui, Behavioral Modeling and Predistortion of Wireless Transmitters, New York: Wiley, 2015.

[16] L. Guan, A. Zhu, "Green Communications: Digital Predistortion for Wideband RF Power Amplifiers", IEEE Microw. Mag., nov./dec. 2014.

[17] A. Hekkala, M. Lasanen, L.C. Vieira, N.J. Gomes, and A. Nkansah, "Architectures for joint compensation of RoF and PA with nonideal feedback", In Vehicular Technology Conference (VTC 2010-Spring), 2010.

[18] L. C. Vieira, N. J. Gomes, A. Nkansah, F. van Dijk, ”Behavioral modeling of radio-over-fiber links using memory polynomials", Proc. IEEE Int. Topical Meeting on Microwave Photonics, Montreal, Canada, 2010.

[19] A. V. Cartaxo, T. M. Alves, F. W. Carvalho, and J. A. Morgado, "Linearization techniques of electro-optical converters for multi-format OFDM-based PONs", In 14th International Conference on Transparent Optical Networks (ICTON), 2012.

[20] A. Hekkala, M. Hiivala, M. Lasanen, J. Perttu, L. C. Vieira, N. J. Gomes, and A. Nkansah, "Predistortion of radio over fiber links: algorithms, implementation, and measurements", IEEE Trans. on Circ. and Syst. - I, 59(3), 664-672, 2012.

[21] Y. Pei, K. Xu, J. Li, A. Zhang, Y. Dai, Y. Ji, and J. Lin, "Complexity-reduced digital predistortion for subcarrier multiplexed radio over fiber systems transmitting sparse multi-band RF signals", Optics express, 21(3), 3708-3714, 2013.

[22] H. Chen, J. Li, K. Xu, Y. Pei, Y. Dai, F. Yin, and J. Lin, "Experimental investigation on multi-dimensional digital predistortion for multi-band radioover-fiber systems", Optics express, 22(4), 4649-4661, 2014.

[23] Y. Zhang, J. Li, H. Chen, C. Yin, Y. Dai, F. Yin, and K. Xu, "Clip-and-Filter-Based Crest Factor Reduction and Digital Predistortion for WLAN-Over-Fiber Links", IEEE Photon. Technol. Lett., 26(23), 2315-2318, 2014.

[24] F. Carvalho, and A. Cartaxo, "Broad baseband nonlinear distortion mitigation using digital pre-and post-distortion in OFDM-based WDM LR-PON", Optics express, 23(6), 7062-7074, 2015.

[25] L. C. Vieira, and N. J. Gomes, "Clipping and predistortion for compensation of OFDM-Radio over Fiber link distortion", IEEE International Conference on Communications, 2015.

[26] G. Khanna, B. Spinnler, S. Calabr, E. De Man, and N. Hanik, "Robust Adaptive Pre-Distortion Method forOptical Communication Transmitters", IEEE Photon. Technol. Lett., vol. 28, no. 7, pp. 752-759, 2016.

[27] Z. Liu, M. A. Violas, and N. B.Carvalho, "Digital predistortion for RSOAs as external modulators in radio over fiber systems", Optics express, 19(18), 17641-17646, 2011.

[28] Y. Bao, Z. Li, J. Li, X. Feng, B.O. Guan, and G. Li, "Nonlinearity mitigation for high-speed optical OFDM transmitters using digital pre-distortion", Optics express, 21(6), 7354-7361, 2013.

[29] H. Moon, R. Sedaghat, "FPGA-based adaptive digital predistortion for radio-over-fiber links", Microprocessors and Microsystems, 30(3), pp. 145-154, 2006.

[30] X. N. Fernando, A. B. Sesay, "Higher order adaptive filter based predistortion for nonlinear distortion compensation of radio over fiber links", IEEE International Conference on Communications, 2000.

[31] L. C. Vieira, N. J. Gomes, A. Nkansah, and F. van Dijk, "Study of complex-envelope behavioral models for radio-over-fiber link nonlinearities", In Global Communications Conference (GLOBECOM), 2012 IEEE (pp. 3098-3103).

[32] P. W. Berenguer, M. Nlle, L. Molle, T. Raman, A. Napoli, C. Schubert, J. K. Fischer, "Nonlinear Digital Pre-distortion of Transmitter Components", J. of Lightw. Technol., 34(8), 1739-1745, 2016.

[33] A. K. Vyas, N. Agrawal, "Development of hybrid envelop memory polynomial based predistorter for RoF system", Optik-Int. Journal for Light and Electron Optics, 127(11), pp. 4768-4773, 2016.

[34] E. Agrell et al., "Roadmap of optical communications", Journal of Optics, Volume 18, Number 6, 2016.

[35] W. H. Press, S. A. Teukolsky, W. T. Vetterling, B. P. Flannery, Numerical Recipes: The Art of Scientific Computing (3rd ed.), New York: Cambridge University Press, 2007.

[36] C. C. Paige, M. A. Saunders, 'LSQR: An algorithm for sparse linear equations and sparse least squares", ACM Trans. on Mathematical Software, 8 (1), pp. 43-71, 1982. 